\title{
Design of Control System of 7.5KW Soft Switch Charger
}

\author{
Huiqin CHEN $^{1, \mathrm{a}}$ ZHaoyan XIE $^{2}$ \\ ( ${ }^{1}$ Southeast University Chengxian College, Nanjing ,Jiangsu,China; ${ }^{2}$ Institute of automation Shandong \\ academy of sciences, Jinan, Shandong,China)
}

('41024257@qq.com)

Keywords: charger ADUC831 UCC3895PWM control system

Abstract: Charger control system is the core components of soft switch charger,its performance determines the technical level of the charger.This paper is based on The ADuC831 MCU and UCC3895PWM, Studies the control system of 7.5KW soft switch charger,designs the hardware system and software system. The system has reliable performance and advanced technology, has a broad market prospects.

\section{Introduction}

In recent years, with the rapid development of electric vehicle industry, the market demand of all kinds of automobile charger is more and more big. Because of the large volume, low efficiency and narrow adjustable range of the output of the ordinary automobile charger, it is difficult to meet the demand of the modern electric vehicle.

Soft switch—_ the new technology of power electronics is applied to the battery charger, which improves the performance of battery power supply. The charger control system is the core component of the soft switch charger, and its performance determines the technical level of the charger. This study is based on a certain type of soft switch $7.5 \mathrm{KW}$ car charger, the charger design control system based on ADUC831 microcontroller, solve the charger intelligent monitoring and automatic control, optimize the charger charging process, improve battery life and capacity, field operation test, reliability and practicability is very high.

\section{System hardware design}

The structure of the control system is shown in Figure 1, including the following functional modules. (1) analog acquisition circuit. (2) digital output circuit. (3) ADUC831 MCU system. (4) protection circuit. (5) regulator, according to the given signal and feedback signal to adjust the PI, the output voltage signal to the pulse generator circuit. (6) the current sharing circuit is used to realize the current sharing function in the parallel connection of multiple chargers. (7) pulse generating circuit.

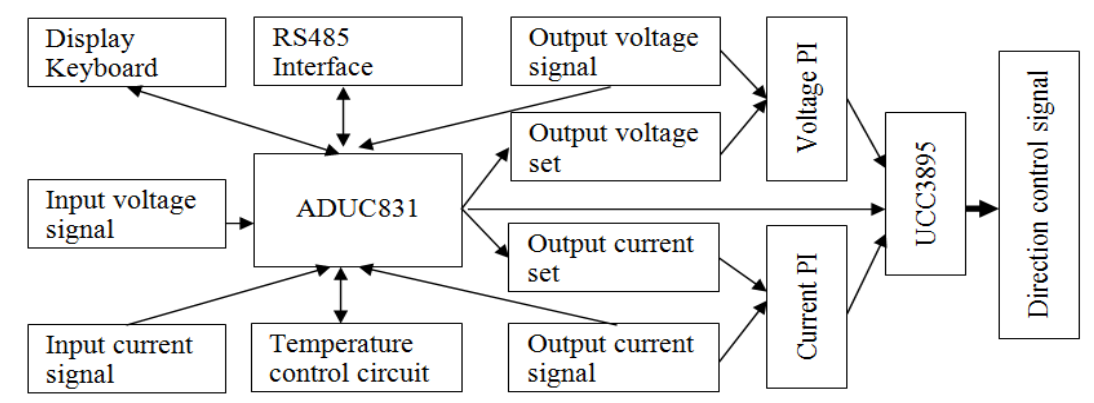

Fig. 1 Structure block diagram of the system 


\subsection{ADUC831 MCU minimum system}

The control system of charger is based on ADI 8 of the company, which is the core of ADUC831. ADUC831 is responsible for the collection and monitoring of the peripheral signal, the control of the output voltage and current of the charger, as well as the protection function of the whole charger system. Charger control circuit is mainly used in the following modules: (1) ADC module. ADC0, ADC1, ADC2 three channels are used to collect the output voltage, output current and internal temperature signal of the charger. (2) DAC module. SCM DAC0, DAC1, respectively, to provide charger output voltage and output current of a given value. (3) timer 0. MCU timer module, a working time of 20 milliseconds. (4) UART module. The UART module is used to receive the control signal of the host computer and send the working state of the charger.

\section{2 detection circuit design}

In the work of the charger need to detect a number of switches and analog, after the signal conditioning circuit, coupled to the microcontroller through the optocoupler to judge and protect. The DC bus voltage is rectified by the diode, and the low voltage signal is generated by the resistance voltage divider; The low voltage signal and the reference voltage are compared with the comparator LM393 to generate the overvoltage, undervoltage and phase missing signals; Overvoltage, undervoltage and lack of phase signal and then through the optocoupler, isolated transmission to the microcontroller.

First, the output voltage of the charger is divided into the LM358 amplifier to be amplified and followed by the LM393 amplifier, and the output voltage and the undervoltage signal are generated by comparing the signal and the reference voltage obtained by the comparator. In order to prevent the external interference from the single chip microcomputer system, the system is unstable, so it is necessary to do signal processing.

The output current signal is sampled by the current sampling resistance of $2.5 \mathrm{ohm}$, and the output current is sampled. The sampled signal is processed by the high precision amplifier OP07 to obtain the analog signal which can be processed by the microcontroller; Singlechip through the A/D converter to obtain the output current value to determine whether the output over-current, resulting in the output over-current protection signal.

\subsection{Circuit design of pulse generator}

As the charger converter for the phase shifted full bridge converter, the design of the UCC3895 control chip. Application circuit schematic shown in figure 2.

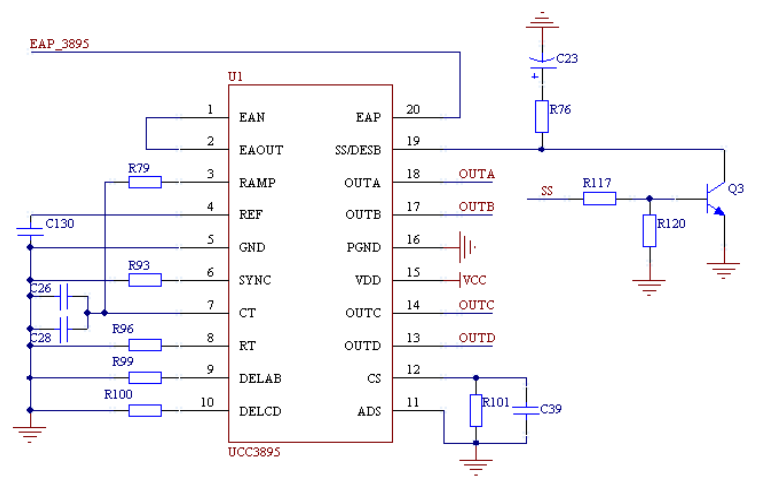

Fig. 2 circuit diagram of UCC3895

In figure 2, the ADS pin function is to set the maximum and minimum values of the output delay dead zone. The ADS pin can change the voltage on the DELAB and DELCD pins by changing the relationship between the (1) and change the output delay.

$$
\mathrm{V}_{\mathrm{DEL}}=\left[0.75 \times\left(\mathrm{V}_{\mathrm{CS}}-\mathrm{V}_{\mathrm{ADS}}\right)\right]+0.5 \mathrm{~V}
$$

The CS pin is the inverting input of the current sense comparator, the noninverting input of the ADS amplifier and the overcurrent comparator. When the circuit operates in the peak current mode, 
the pin signal can realize the function of current limiting by cycle by cycle. The fixed dead time voltage control mode is adopted to connect the CS and ADS pins to the ground, VDEL $=0.5 \mathrm{~V}$. The RAMP pin of the chip is set to operate in the voltage control mode, which is connected to the oscillator output CT. The operating frequency of the synchronous oscillator is determined by the timing capacitor CT and timing resistor RT. Capacitor CT generates a sawtooth wave under the integral action of the current 8 level $\mathrm{T}$ controlled by RT. The period of oscillation can be approximately obtained by the formula (2), and the CT and RT values of the phase shifted pulses generated by the switching frequency $20 \mathrm{KHz}$ are calculated. When $\mathrm{T}=50 \mathrm{uS}=2 \mathrm{Tosc}$, we can calculate the RT x CT=240uS, take $C T=1.2 \mathrm{nF}$, can get $\mathrm{RT}=200 \mathrm{~K}$.

$$
\text { T_OSC }=5 \text { RT } \times \text { CT } / 48+120 \mathrm{~ns}
$$

The DELAB and DELCD pins are used to control dead time. The dead time delay with two tubes of the bridge arm by equation (3) to determine the dead time, design Tdelay $=5 \mathrm{uS}$, Rdel=200K can be obtained.

$$
\mathrm{T}_{\mathrm{DELAY}}=\frac{\left(25 \times 10^{-12}\right) \mathrm{R}_{\mathrm{DEL}}}{\mathrm{v}_{\mathrm{DEL}}}+25 \mathrm{~ns}
$$

REF pin is the output of a voltage reference source, which can output $5 \mathrm{~mA}$. The EAN and EAP pins are the inverting input of the differential amplifier and the input of the same direction. The EAN is designed to be grounded, and the EAP is connected to the output of the PI regulator. EAOUT pin is the output of the differential amplifier. SS/DISB pin control soft start and lock pulse pin. When the voltage is lower than $0.5 \mathrm{~V}$, the chip will immediately block the pulse. All pulse output pins are low. When the SS/DISB is higher than $0.5 \mathrm{~V}$, the output voltage difference amplifier box, can play the role of soft start.

In Figure 2, an electrolytic capacitor C23 is connected between the SS/DISB pin and the ground, and a triode Q3 and a resistor R76 are connected in series with the capacitor. The SS/DISB is connected with the constant current source, and the output current of the constant current source is Irt. When the shutdown, the transistor is turned on, then the SS/DISB voltage is $0 \mathrm{~V}$, the pulse output pin is low state. When activated, the processor provides a start signal, the protection circuit in the absence of other fault signal will be given a low level to the base of the transistor, the transistor off. The constant current source inside the chip begins to charge the capacitor C23 from the Irt current, and the voltage of the SS/DISB rises slowly. The output voltage of the differential amplifier also rises slowly under the clamping action of the SS/DISB, and the phase shift angle increases gradually, then the output voltage of the charger is gradually increased, and the soft start of the charger is realized. When the charger malfunction of the protection circuit will output a high level to S5 the base of the triode R76 to C23 by capacitance discharge, the phase angle decreases, when the pin voltage is lower than $0.5 \mathrm{~V}$, blockade pulse. In order to quickly stop when the fault occurs, we use the resistance R76 resistance is very small, the reason is that the electricity on the capacitor quickly. OUTA, OUTB, OUTC, OUTD is the four pulse output, and the corresponding relationship between the main circuit IGBT shown in figure 3.

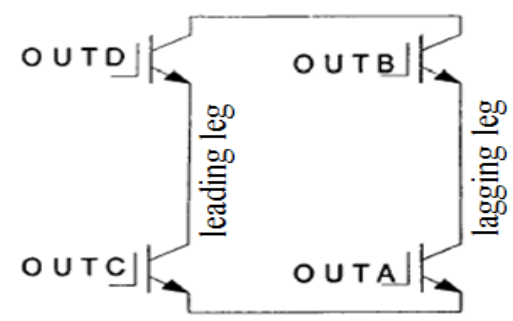

Fig. 3 Corresponding relation graph 


\subsection{Controller hardware circuit design}

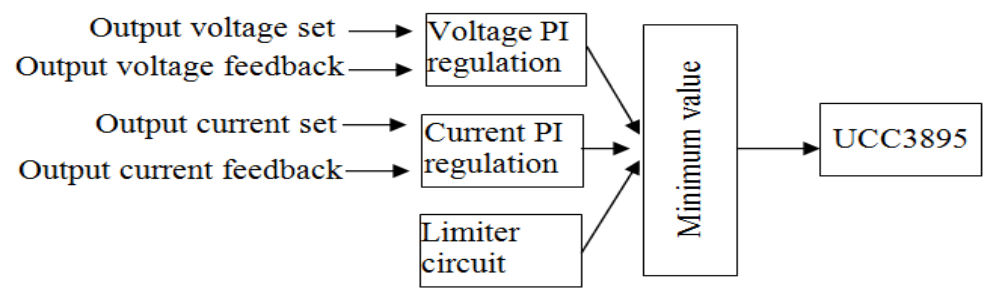

Fig. 4 control block diagram

Adjust the control diagram shown in Figure 4, the actual output voltage and output current will be given the value of simulation operation and feedback regulation of PI obtained by A/D conversion and filtering value, two PI output voltage, output current regulator is a parallel structure, the minimum output from two PI regulator in. By adjusting the output of the regulator, automatic constant current charging and constant voltage charging two charging stages of conversion. At the beginning of the charging, the output current of the ADUC831 is given from the PWM port and the voltage is given. The given value of the current, the constant current charge, the current value of $\mathrm{I}=0.1 \mathrm{C}$. Given voltage, constant voltage charging, voltage $\mathrm{U}=2.4 \mathrm{Fxn}$ value.

Start charging, charging voltage of less than U, voltage PI saturation output. At this time only the current PI, charging constant current charging, charging voltage gradually increased. When the charging voltage exceeds $\mathrm{U}$, the voltage PI is out of saturation. When the output of the voltage regulator is lower than the current regulator, the output value takes the value of the voltage regulator, and the automatic conversion of the constant current charging to the constant voltage charging is completed Then the current continues to decrease, the current regulator is always in a saturated state, only the voltage PI, charger constant voltage charge. When the ADUC831 detects that the charging current is less than the set down current, the stop command is issued.

\subsection{Controller hardware circuit design}

Signal processing and isolation circuit is driven by the TX-DA962D series of high-power IGBT Beijing luomuyuan company driver board. Circuit diagram shown in figure 5.

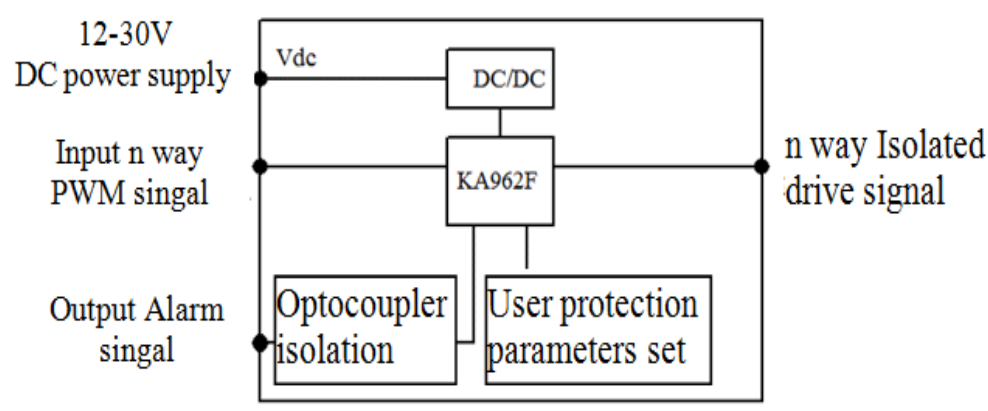

Fig. 5 Circuit diagram of UCC3895

\section{Charger control software design}

The whole system software is designed by modularization. The system includes the initialization module, the output voltage and current adjustment module, the charging state machine module, the output analog detection module, the fault detection module and so on. The specific software structure is shown in Figure 6 and the flow chart is shown in figure 7. 


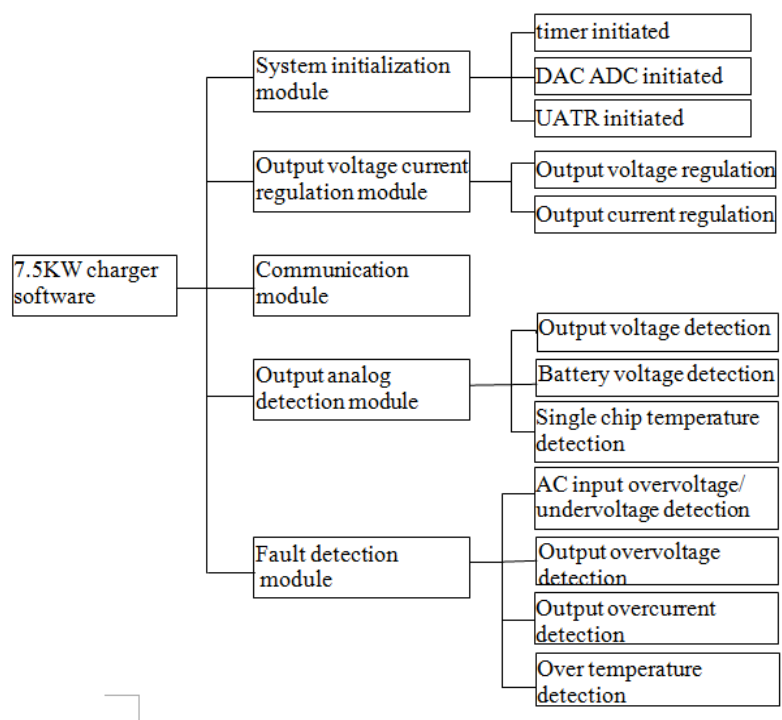

Fig. 6 Software structure diagram

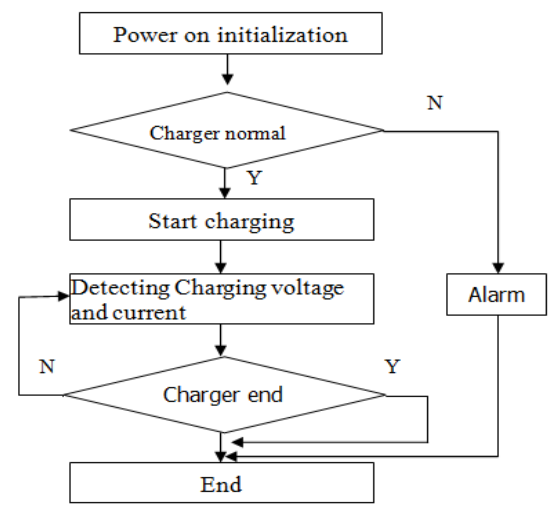

Fig. 7 Systems flowchart

\section{Experimental test}

After the prototype of the $7.5 \mathrm{~kW}$ charger is developed, the system is tested, and the experimental instrument uses the YOKOGAWA DL750 and the test equipment. Figure 8 shows the experimental waveforms for the output voltage $500 \mathrm{~V}$, the output current of $15 \mathrm{~A}$ waveform. CH5 is the CE voltage of the upper arm, $\mathrm{CH} 6$ is the $\mathrm{CE}$ voltage of the lagging arm, $\mathrm{CH} 7$ is the voltage waveform of the inductor, and the $\mathrm{CH} 8$ is the current waveform of the transformer. From the test waveform, the original edge current waveform in the IGBT open and close, the current dropped to 0 , can achieve zero current switch, basically solved the problem of energy loss caused by IGBT trailing current. The test results show that the power factor of the charger 0.954 as shown in Table 1 , the accuracy of the regulator is not more than $\pm 0.5 \%$, as shown in Table 2 , steady flow accuracy of not more than $\pm 1 \%$, as shown in table.

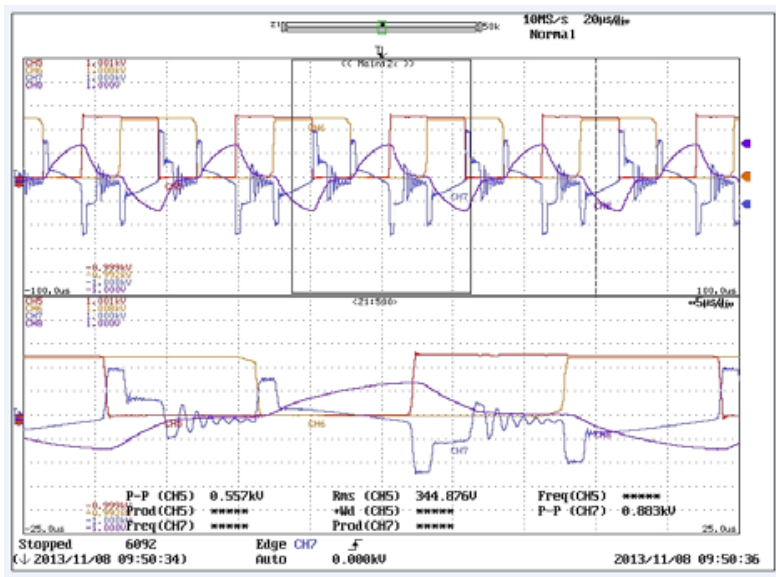

Fig.8 Waveform diagram

Table 1 Charger power factor

\begin{tabular}{|c|c|c|c|c|c|}
\hline Charging station name & 7.5KW charger & Number & 001 & Charger model & ZDH-001 \\
\hline Rated input voltage & $380 \mathrm{~V}$ & Nominal Output voltage & $500 \mathrm{~V}$ & Nominal Output current & $15 \mathrm{~A}$ \\
\hline \multirow[t]{2}{*}{ Input voltage value } & $380 \mathrm{~V}$ & Output voltage setting & $470 \mathrm{~V}$ & Output current setting & $12 \mathrm{~A}$ \\
\hline & & Actual output voltage & $468.690 \mathrm{~V}$ & Actual output current & $11.970 \mathrm{~A}$ \\
\hline AC input $\mathrm{Ua}$ & $378.886 \mathrm{~V}$ & AC input $\mathrm{Ub}$ & $380.012 \mathrm{~V}$ & AC input Uc & $379.839 \mathrm{~V}$ \\
\hline AC input Ia & $10.101 \mathrm{~A}$ & AC input Ib & $10.269 \mathrm{~A}$ & AC input Ic & $10.107 \mathrm{~A}$ \\
\hline Active power & $6.374 \mathrm{~kW}$ & Charger efficiency & $88.02 \%$ & power factor & 0.954 \\
\hline
\end{tabular}


Table 2 The precision of AC output

\begin{tabular}{|c|c|c|c|c|}
\hline Input & AC voltage $(\mathrm{V})$ & load current (A) & DC output voltage ( V ) & stabilized voltage precision (\%) \\
\hline & 323 & \multirow{3}{*}{0} & $450.460 \mathrm{~V}$ & \multirow{3}{*}{0.14} \\
\hline & 380 & & $450.590 \mathrm{~V}$ & \\
\hline & 437 & & $450.610 \mathrm{~V}$ & \\
\hline & 323 & \multirow{3}{*}{4.5} & $450.440 \mathrm{~V}$ & \multirow{3}{*}{0.15} \\
\hline & 380 & & $450.620 \mathrm{~V}$ & \\
\hline & 437 & & $450.680 \mathrm{~V}$ & \\
\hline & 323 & \multirow{3}{*}{9} & $449.420 \mathrm{~V}$ & \multirow{3}{*}{0.14} \\
\hline & 380 & & $450.590 \mathrm{~V}$ & \\
\hline & 437 & & $450.610 \mathrm{~V}$ & \\
\hline & 323 & \multirow{3}{*}{13.5} & $449.230 \mathrm{~V}$ & \multirow{3}{*}{-0.17} \\
\hline & 380 & & $449.310 \mathrm{~V}$ & \\
\hline & 437 & & $450.670 \mathrm{~V}$ & \\
\hline
\end{tabular}

Table 3 The precision of current output

\begin{tabular}{|c|c|c|c|}
\hline Input AC voltage ( V ) & DC output voltage $(\mathrm{V})$ & load current ( A ) & stabilized current precision $(\%)$ \\
\hline 323 & \multirow{3}{*}{450} & 3.03 & \multirow{3}{*}{0.00} \\
\hline 380 & & 3.03 & \\
\hline 437 & & 3.03 & \\
\hline 323 & \multirow{3}{*}{500} & 3.03 & \multirow{3}{*}{0.00} \\
\hline 380 & & 3.03 & \\
\hline 437 & & 3.03 & \\
\hline 323 & \multirow{3}{*}{550} & 3.03 & \multirow{3}{*}{0.33} \\
\hline 380 & & 3.02 & \\
\hline 437 & & 3.02 & \\
\hline
\end{tabular}

\section{Conclusion}

This paper takes the ADUC831 microcontroller and UCC3895PWM phase-shift controller as the core, the design of the control system of ZVZCS soft switch charger based on 7.5KW system structure, stability, reliability, performance indicators meet the requirements of practical application, in the new energy vehicle charging facilities and has a broad market prospect.

\section{Reference}

[1] Zheng Yi, Jiang Jiuchun, Zhang Weige, Niu Liyong. Based on the application of 10KW charger design [J]. electrical FB-ZBZCS-PWM converter 2007 (9): 57-59

[2] Wan Wenbin, Xiang Jian, Jia Jianxiong. Development of control system for high power intelligent charger [J]. electric application 2013 (1): 33-36

[3] Zheng Yi.10KW soft switching charger design [D]. Beijing Jiaotong University

[4] Zhang Yanli, Fei Ren. Development of intelligent charger controller for high power battery [J]. North China Electric Power Technology 2001 (5): 41-43

[5] Luo Wei, Xu Wenjing, Liu Sining et al. Design of high power intelligent charger [J]. Journal of Wuhan University of Technology 2012 (12): 712-714

[6] Zhang. Zhenglong portable electric vehicle charger of [D]. Zhejiang Normal University 